\title{
Treatment of advanced stage osteochondrosis dissecans in the adolescent elbow using a hyaloronic acid-based scaffold: a case series of 5 patients
}

\author{
Sebastian Farr ${ }^{1}$ (D) $\cdot$ Matthias Pallamar ${ }^{1} \cdot$ Theresa Eder $^{2} \cdot$ Rudolf Ganger $^{1}$
}

Received: 21 July 2020 / Accepted: 6 January 2021 / Published online: 4 February 2021

(c) The Author(s) 2021

\begin{abstract}
Introduction Osteochondrosis dissecans (OCD) is considered to be one of the main causes for pain, discomfort and morbidity in the pediatric elbow joint. Few treatment options, such as microfracture or autologous transplantation, of osteochondral bone grafts have been described to address advanced OCD. The aim of this retrospective case series is to present preliminary clinical and radiologic findings following advanced stage OCD repair using a novel combination of a hyaluronic acid-based scaffold with autologous iliac crest bone grafting.

Materials and methods Five adolescents, who underwent treatment of OCD (grade 3 or 4 according to Nelson) using a combination technique of defect debridement, transplantation of cancellous iliac crest bone and application of a HYALOFAST ${ }^{\circledR}$ membrane (Anika Therapeutics S.r.L., Italy), were re-assessed using clinical and radiologic examinations (defect diameter, depth, sclerosis, congruency, fragmentation, dissection, radiolucency, growth plate status; MRI) after a minimum of 2 years (mean, 34 months; range, 24-45) postoperatively. Dedicated outcome scores (Numeric Rating Scale [NRS], Pediatric Outcome Data Collection Instrument [PODCI], Mayo Elbow Performance Score [MEPS], and Timmerman-Andrews Score [TIMM] were collected.

Results All patients reported a NRS score of 0. The mean total TIMM, MEPS and PODCI (Global Functioning Scale) scores were 189 (range 165-200), 94 (range, 70-100), and 92 (range 83-98; normative score 47; range 35-55), respectively, indicating good to excellent clinical outcomes. The radiographic analysis showed overall improvements with regard to OCD width and depth reduction $(35 \%,-27-100 \% ; 52 \%, 4-100 \%)$, but full resolution in only 2 of 5 cases. Elbow motion improved slightly after surgery. No complications were noted.

Conclusion This study showed promising clinical short- to mid-term results in adolescent patients with advanced OCD using a novel surgical treatment combination. Radiographic results showed partial healing; hence, residual changes should be monitored over a longer period.
\end{abstract}

Keywords Osteochondrosis dissecans · Osteochondritis · AMIC · Bone graft · Pediatric elbow

\section{Introduction}

Sebastian Farr

sebastian.farr@oss.at; sebastian.farr@meduniwien.ac.at

1 Orthopedic Hospital Speising, Department of Pediatric Orthopaedics and Adult Foot and Ankle Surgery, Medical University Vienna, Speisingerstrasse 109, 1130 Vienna, Austria

2 Medical University Vienna, Vienna, Austria
Osteochondrosis dissecans (OCD) incidence has been reported to be around 2.2 per 100,000 (3.4 per 100,000 for 12- to 19-year-olds) and may be more prominent in athletes, who are particularly prone to repetitive loading in the radiocapitellar joint $[1,2]$. Male gender and extreme obesity were found to be further risk factors for the development of elbow OCD $[1,3]$. To date, current literature indicates that repetitive stress and altered radiocapitellar mechanics are likely the main causes for OCD besides unknown environmental, endocrinologic and genetic factors $[4,5]$. Untreated OCD may progress to degenerative, osteoarthritic-like changes of 
the cartilage [6]. Therefore, thorough evaluation of the painful elbow joint in young children and athletes is mandatory to avoid delayed diagnosis and progression to surgery $[7,8]$.

Several different treatment strategies have been applied in both immature and adult patient cohorts. In general, treatment is guided by the stage of OCD and whether the OCD lesion is stable or unstable. In general, early-stage pediatric OCD (with open growth plates) can be successfully handled by conservative means, such as rest and sports restriction [9]. However, previous reports have shown that $55 \%$ of elbow OCD cases still progress to surgical treatment [8]. The options for therapy-resistant and/or advanced OCD include fragment resection alone [10], arthroscopy with antegrade or retrograde drilling [11-13], fixation of the fragment using bioabsorbable implants $[14,15]$ or osteochondral autografts (OAG $[16,17])$, and corrective osteotomy of the distal humerus $[18,19]$. In cases with OCD grade 4 (loose body), it is unavoidable to create a new subchondral bone stock and cartilage layer in the defect area [20]. This can be achieved by either autologous transplantation of OAG, which has the potential disadvantage of donor site morbidity (e.g. in the knee joint), or by application of tissue scaffolds [21, 22]. The latter have been shown to be effective in achieving a stable, regenerative cartilage-like tissue [22]. Several reports in the adult literature have highlighted the great healing potential of the so-called autologous matrix-induced chondrogenesis (AMIC) technique in the knee and ankle joint [23-25]. Of note, no such reports have so far been published with regard to an immature patient cohort.

The aim of this retrospective case series is to present our preliminary clinical and radiologic findings following advanced stage OCD repair using a novel combination of a hyaluronic acid-based scaffold with autologous cancellous bone grafting. We sought to determine whether this technique will result in radiographic incorporation of the bone grafting and reduction of pain.

\section{Materials and methods}

This retrospective study was approved by the institutional ethics committee and all participants and/or their caregivers gave informed consent for participation. All children and adolescents $(<18$ years of age) who underwent treatment of idiopathic advanced elbow OCD (grade 3 or 4 according to Nelson [26]) using our technique described below between 2016 and 2018 were invited for a single clinical and radiologic follow-up examination at a minimum of one year after the intervention. The technique is reserved for advanced cases which show detached, unstable fragments in radiographs and MRI/CT (grade 3), or loose bodies (grade 4). All cases older than 18 years of age, those with posttraumatic osteochondral lesions and those with incomplete documentation were excluded. All patients were operated by a single fellowship-trained pediatric upper limb surgeon.

The included patients underwent clinical examination (elbow and forearm motion, stability, tenderness radial head/capitellum); furthermore, anterioposterior (AP), lateral, and AP radiographs in $45^{\circ}$ elbow flexion were obtained in a standardized manner. Magnetic resonance imaging (MRI) sequences were obtained preoperatively and during the postoperative course. The following postoperative outcome scores were obtained: pain on the numeric rating scale (NRS; 0-10), Pediatric Outcome Data Collection Instrument (PODCI; parent-reported version; 0-100 [27]), Mayo Elbow Performance Score (MEPS, 0-100 [28]), and TimmermanAndrews Score (TIMM, 0-200 [29]). The PODCI is a scale assessing upper extremity function, transfers and mobility, physical function and sports, comfort, happiness and satisfaction, and expectations for treatment in children. Both parent and adolescent self-report were developed and showed a high reliability and validity [27]. The Timmerman-Andrews Score, which was originally developed for cases with posttraumatic arthrofibrosis, is frequently beeing used in immature cohorts with elbow OCD [16].

The radiologic evaluation aimed to include the following pre- and postoperative OCD features: grade according to Minami [30], Berndt and Harty [31], and Nelson [26]; growth plate status (open/closed); AP and lateral diameter (in mm); AP width and depth (in \% of total epicondylar diameter); congruency of the subchondral bone (flattened, concave, convex); sclerosis (none/yes); dissection (none, partial, complete/loose body); radiolucency (reduced, increased, normal), and fragmentation (none, heterogeneous area, distinct fragmentation). The MRI-related OCD healing after surgery was graded according to Roberts et al. (total score, 0-4) [32].

\section{Surgical technique}

The surgical approach is similar to the one described previously [33]. Briefly, a lateral Kocher approach to the elbow patient is performed with the patient lying in the supine position (Fig. 1a). The fascia is divided and the interval between the extensor digitorum communis and extensor carpi ulnaris is separated to expose the joint capsule. The capsule is then divided and the lateral collateral ligaments are released whenever proper inspection of the capitellum is impaired. The capitellum is thoroughly assessed to search for the affected OCD area, in particular, i.e. cartilage softening, fissures or detached fragments (Fig. 1b, arrow). Any loose boodies are removed instantly, and the radial head is inspected for any concomitant alterations. Then, the OCD defect is debrided (Fig. 1c, arrow). For this reason, the cartilage rim is trimmed to stable edges using the scapel. The subchondral bone layer is sharply curretted 


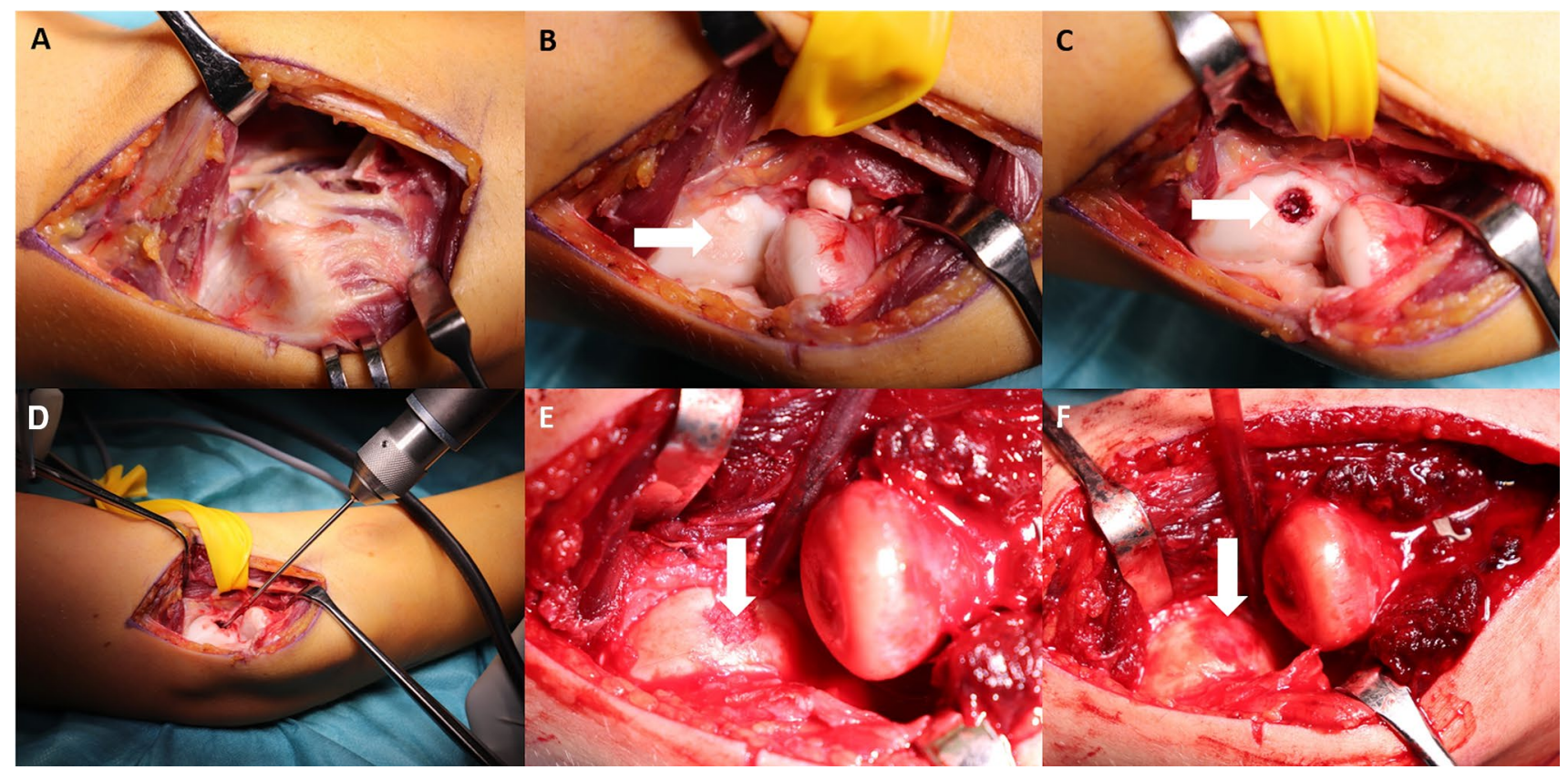

Fig. 1 The surgical approach (a) and technique are presented. After joint inspection and removal of a loose body (b), the defect area is thoroughly curretted to remove all unstable tissue (c, arrow). Then, antegrade drilling is performed and the cancellous bone graft is

to remove the superficial fibrinous layer and all bone material of inferior quality. After the curretage, the OCD area is circumferentially drilled in an antegrade fashion at low drill speed until bleeding is visible (Fig. 1d). Then, autologous cancellous bone as well as bone marrow is harvested from the iliac crest. The cancellous bone is impacted in the defect (Fig. 1e, arrow). It is mandatory to avoid overstuffing of the defect since the reconstructed joint surface should eventually have the same level like the surrounding healthy cartilage (Fig. 1f). Finally, the scaffold (HYALOFAST®; Anika Therapeutics S.r.L., Italien; Fig. 2) is cut to fit the defect size, injected with bone marrow and layed tightly impacted (d, e, arrow). Finally, the scaffold is cut to fit the defect, injected with bone marrow aspirate, and applied over the defect (f, arrow). It is held stable using fibrin glue

over the defect (Fig. 1f, arrow). The membrane is structured as a 3-dimensional matrix which releases hyaluronic acid during its degradation. Fibrin glue is used to stabilize the membrane (Fig. 1f). The lateral collateral ligaments are repaired with non-absorbable sutures whenever necessary. After capsular repair and skin closure, a long arm cast is applied for 3 weeks. Thereafter, protected exercises are allowed using an elbow orthosis (Epico ROMs; medi GmbH \& Co. KG; Bayreuth, Germany) and occupational therapy. Full loadbearing and manual activity is allowed 3 months postoperatively. Sports participation is resumed after 6 months.
Fig. 2 The HYALOFAST® membrane $(2 \times 2 \mathrm{~cm})$ is shown (a). A schematic drawing illustrates the important steps, such as antegrade drilling of the capitulum, to enhance regeneration (white arrows), impaction of cancellous bone graft and application of the membrane (b)

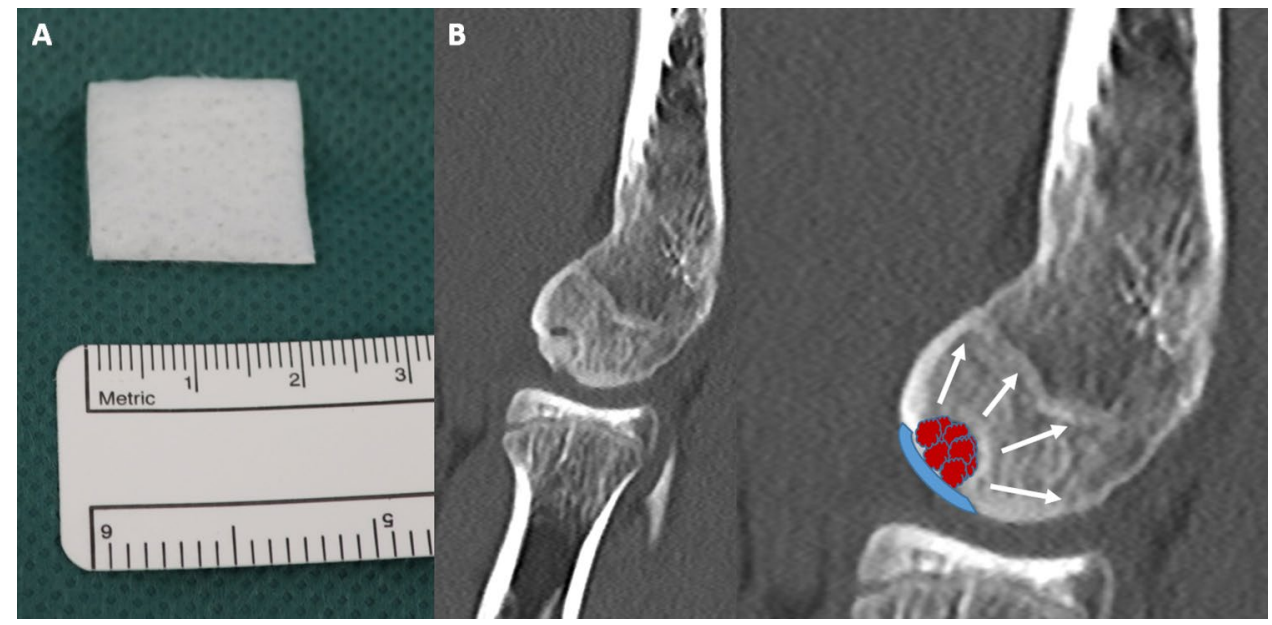




\section{Statistical analysis}

A descriptive calculation of means, minimum and maximum of the main outcome parameters and demographic details were performed. All analyses were performed using SPSS v23.0.

\section{Results}

This series included 5 adolescent patients ( 3 males, 2 females) with a mean age of 13.4 years (range, 12-15) at the time of surgery and 17.3 years at final follow-up (range, 15-20). Hence, the mean follow-up period was 34 months (range, 24-45). Further demographic details are listed in Table 1. Two cases received diagnostic arthroscopy prior to the intervention in the same session to verify fragment instability.

All patients reported an NRS score of 0. Elbow flexion and extension improved from a mean of $132^{\circ}$ (range $110-140$ ) and $-6^{\circ}$ (range -15 to 0 ) preoperatively to $135^{\circ}$ (range 125-140) and $0^{\circ}$ (range $0-0$ ) at latest follow-up. The forearm motion arc improved from a mean of $164^{\circ}$ (range $120-180$ ) to $176^{\circ}$ (range $160-180$ ) after surgery. The mean total TIMM was 189 (range 165-200). TIMM subscales indicated good to excellent outcomes for both subjective (mean, 91; range 65-100) and objective (mean 98; range 90-100) measures. The mean postoperative MEPS was 94 (range 70-100). The mean postoperative PODCI Global Functioning Scale was 92 (range 83-98; normative score 47; range 35-55). All detailed PODCI scales and scores are shown in Table 2.

The radiographic parameters showed a mean decrease of the affected OCD area of $35 \%$ in width (range -27 to $100 \%$ ) and 52\% in depth (range, 4-100\%), respectively. Cases no. 4 and 5 (Fig. 3) showed complete resolution of the reconstructed OCD area while the other cases showed residual findings, such as reduced bone transparency $(n=3)$. A detailed summary of the radiologic findings is highlighted in Table 3.

The MRI findings showed a mean Roberts score of 2.0 (range 1-3) on the scale of 0-4 (Table 4).

\section{Discussion}

Elbow OCD in children and adolescents is a frequent pathology with a high risk for morbidity. In cases with moderate to severe disruption of the subchondral bone, restoration of this affected area and the altered cartilage layer is mandatory. The aim of this case-series was therefore to present our preliminary findings of a new technique which addresses both the degenerated cartilage and bone area.

Early cases of OCD have been shown to sufficiently respond to activity modification and rest [9]. Niu et al. reported that $>50 \%$ of stable OCD cases resolved after a mean period of 8.3 months [34]. Predictors for healing were a smaller OCD area and lack of cyst-like lesions [34]. In turn, a large study of 245 elbows has shown that, among 48 non-surgical cases, radial head enlargement and advanced skeletal age were predictors for a lack of spontaneous healing [35].

In cases where OCD still progresses to increased capitellar involvement, drilling/microfracture or fragment refixation has shown differring results depending on the state of the disease. Matsuura et al. have shown that arthroscopy with concomitant drilling provided enduring and independent results in the long-term indepent of the lesion size [13]. However, in their study, only $6 / 23$ patients (baseball players) had large OCD lesions (defect size $>60 \%$ ) [13]. These results contrast the findings of Ueda et al. who provided evidence that larger OCD lesions, which were simply resected, progressed to osteoarthritis after a mean of 8 years postoperatively [10]. Moreover, another study reported that just $71 \%$ of cases had either clinical or radiographic resolution $(50 \%$ complete resolution on MRI, $62 \%$ clinically non-tender) after drilling of grade IV lesions [12]. Despite a relatively high return-to-any-sports-rate, $43 \%$ still reported mild elbow pain and persisting mechanical symptoms after this surgery [12]. In contrast, Takeba et al. and Hennrikus et al. reported that, in unstable but still in situ lesions, fragment refixation can provide a reasonable healing percentage with good outcomes for the majority of children $[14,15]$.

As a consequence of the findings described above, many authors tend to replace the subchondral, malvascularized

Table 1 Demographic details

\begin{tabular}{llllllllll}
\hline Case & Sex & Side & Age & BMI & Minami & $\begin{array}{l}\text { Berndt } \\
\text { and Harty }\end{array}$ & Nelson & $\begin{array}{l}\text { Sports Par- } \\
\text { ticipation }\end{array}$ & Additional Surgery \\
\hline 1 & F & R & 13 & 18 & III & IV & IV & Yes & None \\
2 & F & L & 14 & 19 & II & III & III & Yes & None \\
3 & M & R & 12 & - & I & II & III & Yes & Arthroscopy \\
4 & M & R & 15 & 20 & III & III & III & Yes & Arthroscopy \\
5 & M & R & 13 & 19 & III & IV & IV & Yes & None \\
\hline
\end{tabular}

M, male; F, female; R, right; L, left; OCD, osteochondrosis dissecans; BMI, body mass index 


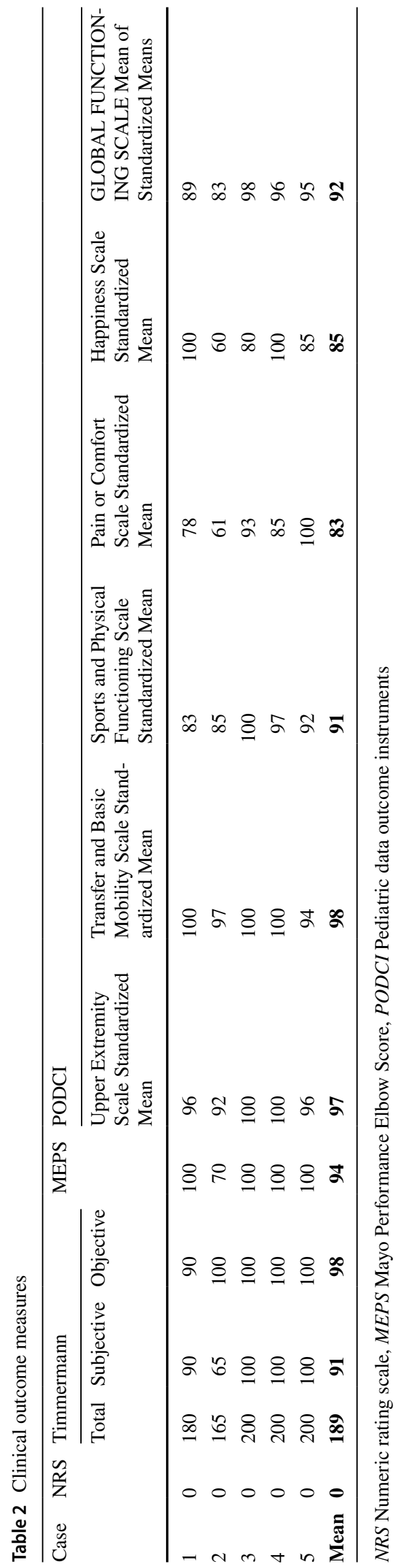

bone in advanced OCD stages (e.g. grades III to IV) using osteochondral bone grafts $[16,17,36,37]$. This can provide additional healthy hyaline cartilage as new joint surface tissue, either transplated as a single bone plug or mosaicplasty. A host of different reports exist showing that favorable results can be achieved with the OAG technique. Bae et al. reported significant improvements in elbow motion, 93\% graft incorporation into the recipient site, and no complications thereafter [16]. As compared to Bae et al. and most other authors who used femoral trochlear autografts, Sato and colleagues reported excellent outcomes after transplantation of costal OAG [17]. Oshiba et al., who used multiple bone plugs for mainly early lesions (ICRS grade I and II; Minami stage I and II) reported complete radiological healing in 8 of 11 cases; however, healing needed up to 24 months to become evident in MRI [16]. Another study has, however, shown that large-size defects, which span from the lateral to the central articular portion, may not heal as well as central lesions after OAG transplantation [36, 37].

The harvest of autologous bone plugs is, however, not without the risk of complications and morbidity [21]. Up to $13 \%$ of patients reported knee symptoms after OAG harvest. Therefore, many patients, especially those involved in sports participation, are in our experience rather reluctant to agree to harvest tissue of their healthy knee. This was one of the reasons for our group to look for alternative techniques to repair the capitellum.

We thus aimed to repair the defect area by thorough debridement (curretage of unstable, softened bone tissue), drilling to enhance re-vascularization, and autologous iliac crest cancellous bone graft infill. The graft harvest is usually very well tolerated since it is performed via a $1 \mathrm{~cm}$ miniincision with a bone punch. The donor site morbidity in this area is more appealing for many patients as compared to the knee joint. The application of the scaffold membrane is easy to do, and already proved to be efficacious in other past reports [23-25]. We additionally prefer to add iliac crest blood to enhance stem-cell-related ingrowth.

The results of our technique were so far quite favorable with regard to clinical improvement. All five patients reported no symptoms at all. One case, a professional skier, experienced residual pain during heavier activities which eventually resolved two years after the surgery. The good outcomes are supported by good to excellent Mayo Elbow Performance, Timmerman Andrews and PODCI scores, respectively. In contrast, radiological findings were moderate. We observed an expected decrease of the OCD area at follow-up, but no full radiologic healing was evident in native radiographs in $3 / 5$ cases. This was, however, not surprising, since the cancellous bone graft may not have the same structure and architecture of the native capitellar bone. However, several partial improvements, such as 

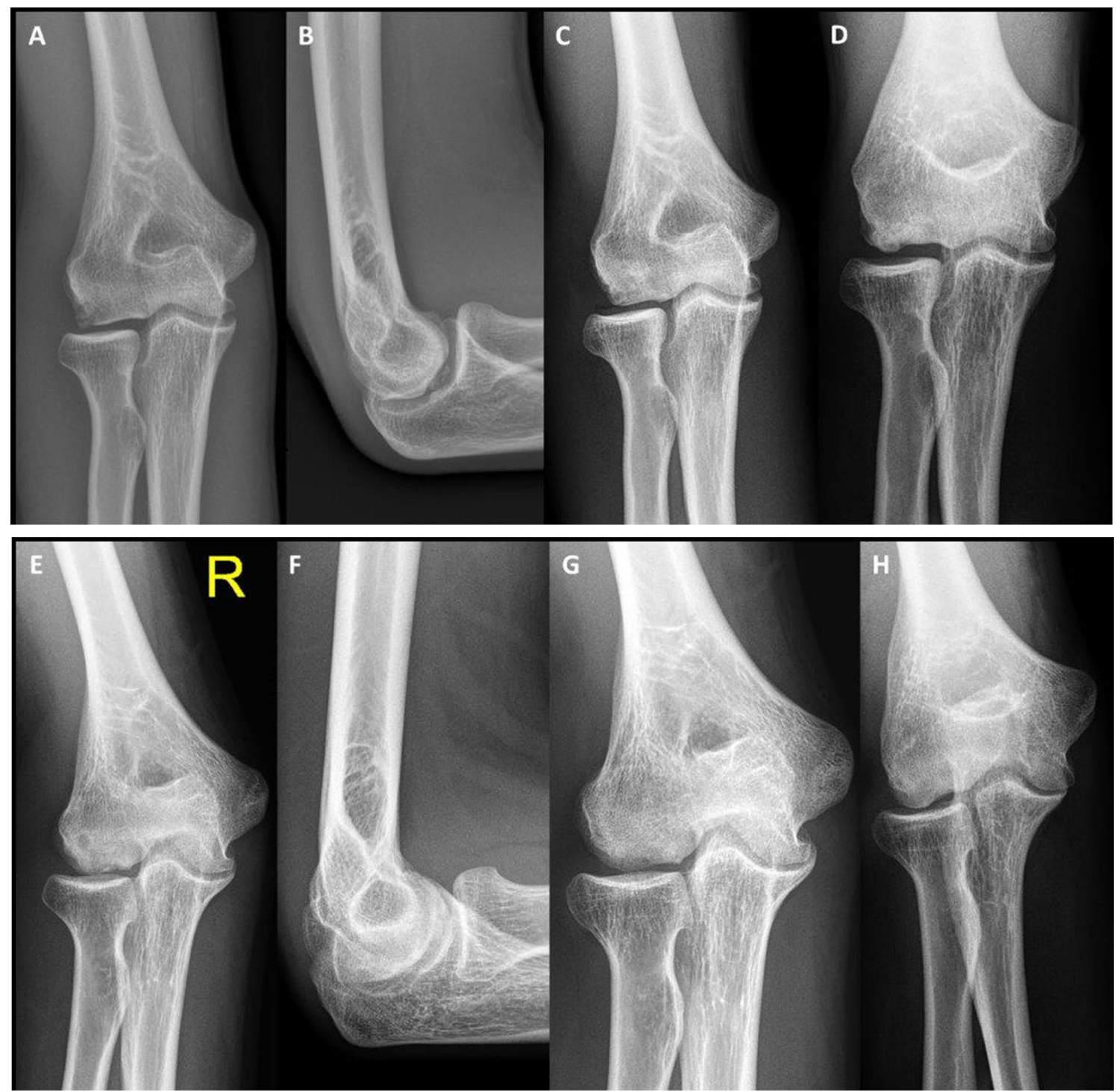

Fig. 3 Two OCD cases are presented. Case 4 revealed a lateral sided defect (a) with a detached fragment (b) which showed good resolution after surgery (c). However, $45^{\circ}$ AP views confirmed some irregularities, likely resembling the cancellous graft (d). Case 5 showed a severe affection of the entire condyle (e,f) but also healed well without any residual symptoms. Postoperative images revealed a homogeneous situation, however, the subchondral area was still concave and slightly hypointense $(\mathbf{g}, \mathbf{h})$

Table 3 Radiographic Outcome and Details

\begin{tabular}{llllllllll}
\hline Case & $\begin{array}{l}\text { Initial } \\
\text { Physis } \\
\text { Status }\end{array}$ & Final Physis Status & $\begin{array}{l}\text { Width } \\
\text { Improve- } \\
\text { ment }\end{array}$ & $\begin{array}{l}\text { Depth } \\
\text { Improve- } \\
\text { ment }\end{array}$ & Final Congruency & Final Sclerosis & $\begin{array}{l}\text { Final } \\
\text { Dissec- } \\
\text { tion }\end{array}$ & $\begin{array}{l}\text { Final Transparency } \\
\text { Final } \\
\text { Fragmen- } \\
\text { tation }\end{array}$ \\
\hline 1 & Closed & Closed & $-9 \%$ & $\mathbf{4 \%}$ & Convex & No & No & Normal \\
2 & Closed & Closed & $\mathbf{1 0 \%}$ & $\mathbf{7 \%}$ & Convex & No & No & Reduced & No \\
3 & Open & Almost closed & $-27 \%$ & $\mathbf{4 7 \%}$ & Convex & No & No & Reduced & No \\
4 & Closed & Closed & $\mathbf{1 0 0 \%}$ & $\mathbf{1 0 0} \%$ & Convex & No & No & Normal & No \\
5 & Open & Closed & $\mathbf{1 0 0}$ & $\mathbf{1 0 0} \%$ & Concave & No & No & Normal \\
\hline
\end{tabular}

Improvements are highlighted in bold 
Table 4 MRI findings

\begin{tabular}{llllll}
\hline Case & $\begin{array}{l}\text { Surface Integrity } \\
\text { and contour }\end{array}$ & $\begin{array}{l}\text { Cartilage signal in } \\
\text { graft region }\end{array}$ & $\begin{array}{l}\text { Cartilage } \\
\text { thickness }\end{array}$ & $\begin{array}{l}\text { Changes in } \\
\text { underlying bone }\end{array}$ & Total score* \\
\hline 1 & 0 & 0 & 0 & 1 & 1 \\
2 & 0 & 0 & 1 & 1 & 2 \\
3 & 0 & 1 & 1 & 1 & 3 \\
4 & 0 & 1 & 1 & 0 & 2 \\
5 & 0 & 0 & 1 & 1 & 2 \\
\hline
\end{tabular}

$1=$ normal or near normal, $0=$ abnormal

*Aggregate score (4 points maximum possible)

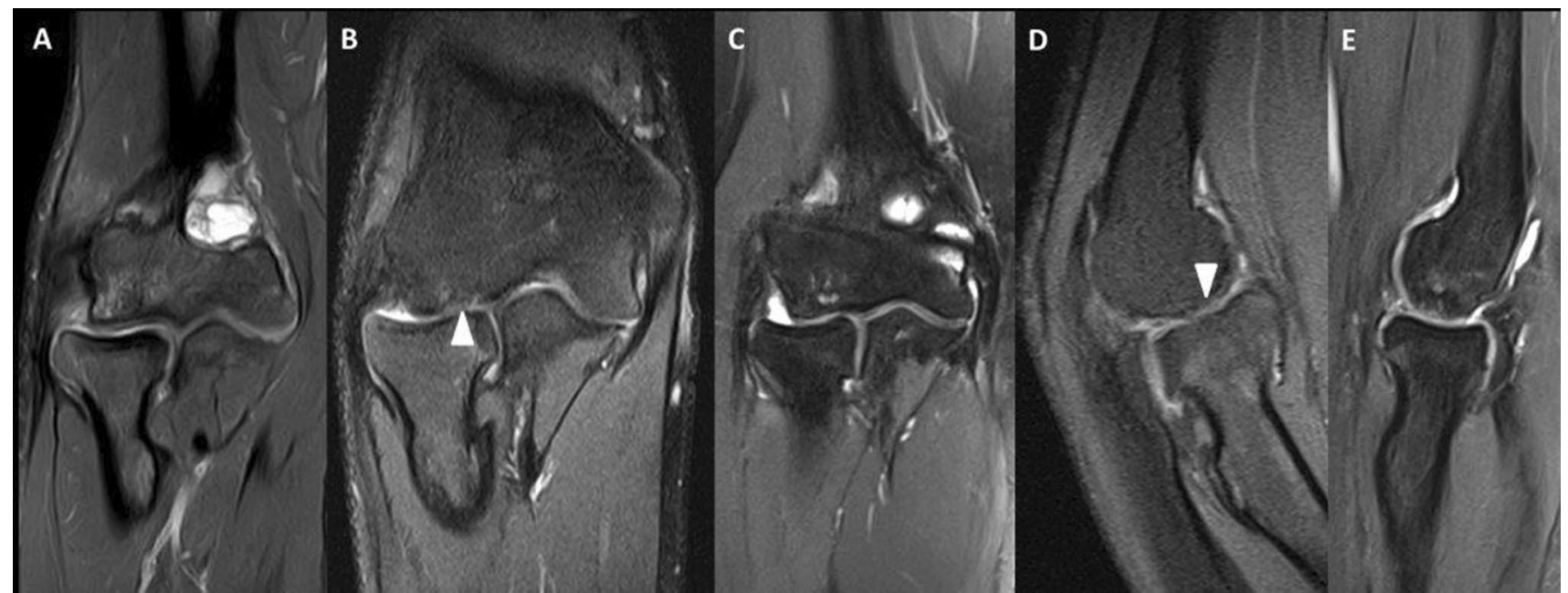

Fig. 4 The postoperative MRI sequences of cases $4(\mathbf{a}), 5(\mathbf{b}, \mathbf{c})$ and $2(\mathbf{d}, \mathbf{e})$ are shown. Reduction of OCD-related edema is visible in all cases. Fibrous regenerate cartilage is highlighted in B and D (arrowhead)

a changes of the subchondral congruency from "flattened" to "convex" (in 2 cases), reduction of sclerosis (in 2 cases) and normalization of transparency and fragementation (in 1 case each) were observed. In regard to MRI findings, previous studies have shown that signs of the disease may still be visible up to 24 months after surgery [36]. Our postoperative MRIs showed lack of subchondral and capitellar bone marrow edema with, however, expected irregularities next to the healthy hyaline cartilage as a sign of fibrous regenerate tissue in the scaffold area (Fig. 4).

The only comparable study, published by Guerra et al., reported on the outcomes following bone marrow-derived cell transplantation in 3 adolescents [22]. In this technique, platelet-rich fibrin was used to enhance local regeneration after clearing of the lesion. All patients reported clinical improvements and resumed sports after 9 months. MEPS improved from 78.3 to 93.3 and the Oxford Elbow Score from 40.0 to 47.6. As seen in our current study, continuous healing with regeneration tissue was seen over time.

The limitation of this study is the small study cohort and the preliminary, mid-term character of our findings.
Furthermore, no alternative technique was included to serve as controls and the radiographs were rated by a single observer (treating surgeon). It remains to be determined whether the results are durable over a longer period and whether radiographic healing may advance over time. We did not include a standardized MRI protocol in our follow-up evaluation and hence, MRIs were obtained at different time points (10-32 months) postoperatively.

In summary, the findings of our novel technique showed promising results in adolescent patients with severe OCD. Due to its relevant advantages, such as the possibility to reconstruct the defect height exactly as needed longer follow-up will be pursued to confirm its suitability as an alternative technique to OAG.

Acknowledgments Open access funding was provided by the Medical University of Vienna.

Author contributions All authors contributed to the study conception and design. Material preparation and data collection was performed by SF, and TE. Data analysis was performed by SF. The first draft of the 
manuscript was written by SF. MP, and RG, revised the manuscript. All authors approved the final manuscript.

Funding Open Access funding provided by Medical University of Vienna. The authors received no financial support for the research, authorship, and/or publication of this article.

\section{Compliance with Ethical Standards}

Conflict of interest Rudolf Ganger, M.D. has received honoraria from Smith\&Nephew, Inc. (Memphis, TN) and OrthoPediatrics Corp. (Warsaw, IN) outside the submitted work. Sebastian Farr, M.D. has received honoraria from Orthofix SRL (Verona, IT) outside the submitted work. All other authors declared no potential conflicts of interest with respect to the research, authorship, and/or publication of this article.

Ethical approval All procedures performed in studies involving human participants were in accordance with the ethical standards of the institutional research committee and with the 1964 Helsinki declaration and its later amendments or comparable ethical standards.

Open Access This article is licensed under a Creative Commons Attribution 4.0 International License, which permits use, sharing, adaptation, distribution and reproduction in any medium or format, as long as you give appropriate credit to the original author(s) and the source, provide a link to the Creative Commons licence, and indicate if changes were made. The images or other third party material in this article are included in the article's Creative Commons licence, unless indicated otherwise in a credit line to the material. If material is not included in the article's Creative Commons licence and your intended use is not permitted by statutory regulation or exceeds the permitted use, you will need to obtain permission directly from the copyright holder. To view a copy of this licence, visit http://creativecommons.org/licenses/by/4.0/.

\section{References}

1. Kessler JI, Jacobs JC Jr, Cannamela PC et al (2018) Demographics and epidemiology of osteochondritis dissecans of the elbow among children and adolescents. Orthop J Sports Med 6(12):2325967118815846. https://doi.org/10.1177/2325967118 815846

2. Matsuura T, Iwame T, Suzue $\mathrm{N}$ et al (2019) Cumulative incidence of osteochondritis dissecans of the capitellum in preadolescent Baseball players. Arthroscopy 35:60-66. https://doi.org/10.1016/j. arthro.2018.08.034

3. Kessler JI, Jacobs JC Jr, Cannamela PC et al (2018) Childhood obesity is associated with osteochondritis dissecans of the knee, ankle, and elbow in children and adolescents. J Pediatr Orthop 38:e296-e299. https://doi.org/10.1097/BPO.0000000000001158

4. Jarrett DY, Walters MM, Kleinman PK (2016) Prevalence of capitellar osteochondritis dissecans in children with chronic radial head subluxation and dislocation. AJR Am J Roentgenol 206:1329-1334. https://doi.org/10.2214/AJR.15.15513

5. Matsuura T, Wada K, Suzue N et al (2017) Bilateral osteochondritis dissecans of the capitellum in fraternal twins: a case report. JBJS Case Connect 7:e44. https://doi.org/10.2106/JBJS. CC. 16.00203

6. Kusumi T, Ishibashi Y, Tsuda E et al (2006) Osteochondritis dissecans of the elbow: histopathological assessment of the articular cartilage and subchondral bone with emphasis on their damage and repair. Pathol Int 56:604-612. https://doi.org/10.111 1/j.1440-1827.2006.02015.x

7. Cheng C, Milewski MD, Nepple JJ et al (2019) Predictive role of symptom duration before the initial clinical presentation of adolescents with capitellar osteochondritis dissecans on preoperative and postoperative measures: a systematic review. Orthop J Sports Med 7:2325967118825059. https://doi.org/10.1177/2325967118 825059

8. Weiss JM, Nikizad H, Shea KG et al (2016) The incidence of surgery in osteochondritis dissecans in children and adolescents. Orthop J Sports Med 4:2325967116635515. https://doi. org/10.1177/2325967116635515

9. Mihara K, Tsutsui H, Nishinaka N et al (2009) Nonoperative treatment for osteochondritis dissecans of the capitellum. Am J Sports Med 37:298-304. https://doi.org/10.1177/0363546508324970

10. Ueda Y, Sugaya H, Takahashi N et al (2017) Arthroscopic fragment resection for capitellar osteochondritis dissecans in adolescent athletes: 5- to 12-year follow-up. Orthop J Sports Med 5:2325967117744537. https://doi.org/10.1177/232596711774453 7

11. Arai Y, Hara K, Inoue $\mathrm{H}$ et al (2019) Early clinical results of arthroscopically assisted drilling via the radius in a distal-to-proximal direction for osteochondritis dissecans of the elbow. Orthop J Sports Med 7:2325967119868937. https://doi.org/10.1177/23259 67119868937

12. Lewine EB, Miller PE, Micheli LJ et al (2016) Early results of drilling and/or microfracture for grade IV osteochondritis dissecans of the capitellum. J Pediatr Orthop 36:803-809. https://doi. org/10.1097/BPO.0000000000000575

13. Matsuura T, Iwame T, Suzue N et al (2020) Long-term outcomes of arthroscopic debridement with or without drilling for osteochondritis dissecans of the capitellum in adolescent baseball players: a $\geq 10$-year follow-up study. Arthroscopy. https:// doi.org/10.1016/j.arthro.2020.01.020

14. Hennrikus WP, Miller PE, Micheli LJ et al (2015) Internal fixation of unstable in situ osteochondritis dissecans lesions of the capitellum. J Pediatr Orthop 35:467-473. https://doi. org/10.1097/BPO.0000000000000308

15. Takeba J, Takahashi T, Watanabe S et al (2015) Short-term clinical results of arthroscopic osteochondral fixation for elbow osteochondritis dissecans in teenaged baseball players. J Shoulder Elbow Surg 24:1749-1756. https://doi.org/10.1016/j. jse.2015.07.009

16. Bae DS, Ingall EM, Miller PE et al (2020) Early results of single-plug autologous osteochondral grafting for osteochondritis dissecans of the capitellum in adolescents. J Pediatr Orthop 40:78-85. https://doi.org/10.1097/BPO.0000000000001114

17. Sato K, Iwamoto T, Matsumura N et al (2018) Costal osteochondral autograft for advanced osteochondritis dissecans of the humeral capitellum in adolescent and young adult athletes: clinical outcomes with a mean follow-up of 4.8 years. J Bone Joint Surg Am 100:903-913. https://doi.org/10.2106/JBJS.17.01035

18. Koda H, Moriya K, Ueki M et al (2019) Long-term results of closed-wedge osteotomy of the lateral humeral condyle for osteochondritis dissecans of the capitellum. J Shoulder Elbow Surg 28:e313-e320. https://doi.org/10.1016/j.jse.2019.05.016

19. Ueki M, Moriya K, Yoshizu T et al (2019) Closed-wedge osteotomy of the distal humerus for treating osteochondritis dissecans of the capitellum in young patients. Orthop J Sports Med 7:2325967119876247. https://doi.org/10.1177/2325967119 876247

20. Jones KJ, Wiesel BB, Sankar WN et al (2010) Arthroscopic management of osteochondritis dissecans of the capitellum: mid-term results in adolescent athletes. J Pediatr Orthop 30:813. https://doi.org/10.1097/BPO.0b013e3181c3be83 
21. Matsuura T, Hashimoto Y, Kinoshita T, Nishino K, Nishida Y, Takigami J, Katsuda H, Shimada N (2019) Donor site evaluation after osteochondral autograft transplantation for capitellar osteochondritis dissecans. Am J Sports Med 47(12):2836-2843. https://doi.org/10.1177/0363546519871064

22. Guerra E, Fabbri D, Cavallo M et al (2018) Treatment of capitellar osteochondritis dissecans with a novel regenerative technique: case report of 3 patients after 4 years. Orthop J Sports Med 6:2325967118795831. https://doi.org/10.1177/23259 67118795831

23. Fossum V, Hansen AK, Wilsgaard T et al (2019) Collagencovered autologous chondrocyte implantation versus autologous matrix-induced chondrogenesis: a randomized trial comparing 2 methods for repair of cartilage defects of the knee. Orthop J Sports Med 7:2325967119868212. https://doi. org/10.1177/2325967119868212

24. Steinwachs MR, Gille J, Volz M et al (2019) Systematic review and meta-analysis of the clinical evidence on the use of autologous matrix-induced chondrogenesis in the knee. Cartilage 11:1947603519870846. https://doi.org/10.1177/1947603519 870846

25. Weigelt L, Hartmann R, Pfirrmann C et al (2019) Autologous matrix-induced chondrogenesis for osteochondral lesions of the talus: a clinical and radiological 2- to 8-year follow-up study. Am J Sports Med 47:1679-1686. https://doi.org/10.1177/03635 46519841574

26. Nelson DW, DiPaola J, Colville M et al (1990) Osteochondritis dissecans of the talus and knee: prospective comparison of MR and arthroscopic classifications. J Comput Assist Tomogr 14:804-808. https://doi.org/10.1097/00004728-199009000-00026

27. Daltroy LH, Liang MH, Fossel AH et al (1998) The POSNA pediatric musculoskeletal functional health questionnaire: report on reliability, validity, and sensitivity to change. Pediatric Outcomes Instrument Development Group. Pediatric Orthopaedic Society of North America. J Pediatr Orthop 18:561-571. https://doi. org/10.1097/00004694-199809000-00001

28. Morrey BF, An KN (1993) Functional evaluation of the elbow. In: Morrey BF (ed) The elbow and its disorders. WB Saunders, Philadelphia, pp 74-83

29. Timmerman LA, Andrews JR (1994) Arthroscopic treatment of posttraumatic elbow pain and stiffness. Am J Sports Med 22:230 235. https://doi.org/10.1177/036354659402200213
30. Minami MN, Nakashita K, Ishii S (1979) Twenty-five cases of osteochondritis dissecans of the elbow. Rinsho Seikei Geka 14:805-810

31. Berndt AL, Harty M (1959) Transchondral fractures (osteochondritis dissecans) of the talus. J Bone Joint Surg Am 41:988-1020

32. Roberts S, McCall IW, Darby AJ, Menage J, Evans H, Harrison PE, Richardson JB (2003) Autologous chondrocyte implantation for cartilage repair: monitoring its success by magnetic resonance imaging and histology. Arthritis Res Ther 5:R60-73. https://doi. org/10.1186/ar613

33. Farr S, Abualruz MM, Girsch W (2020) Radial head resection and corium interposition arthroplasty in skeletally immature patients with isolated posttraumatic radial head arthrosis. Tech Hand Up Extrem Surg 24:37-42. https://doi.org/10.1097/BTH.0000000000 000259

34. Niu EL, Tepolt FA, Bae DS et al (2018) Nonoperative management of stable pediatric osteochondritis dissecans of the capitellum: predictors of treatment success. J Shoulder Elbow Surg 27:2030-2037. https://doi.org/10.1016/j.jse.2018.07.017

35. Funakoshi T, Furushima K, Miyamoto A et al (2019) Predictors of unsuccessful nonoperative management of capitellar osteochondritis dissecans. Am J Sports Med 47:2691-2698. https:// doi.org/10.1177/0363546519863349

36. Oshiba H, Itsubo T, Ikegami S et al (2016) Results of bone peg grafting for capitellar osteochondritis dissecans in adolescent baseball players. Am J Sports Med 44:3171-3178. https://doi. org/10.1177/0363546516658038

37. Yamagami N, Yamamoto S, Aoki A et al (2018) Outcomes of surgical treatment for osteochondritis dissecans of the elbow: evaluation by lesion location. J Shoulder Elbow Surg 27:2262-2270. https://doi.org/10.1016/j.jse.2018.08.011

38. Wetzler MJ (2018) Editorial commentary: is the cure worse than the disease? harvesting autologous osteochondral transfer plugs for treatment of lesions of the elbow. Arthroscopy 34:2094-2095. https://doi.org/10.1016/j.arthro.2018.05.010

Publisher's Note Springer Nature remains neutral with regard to jurisdictional claims in published maps and institutional affiliations. 